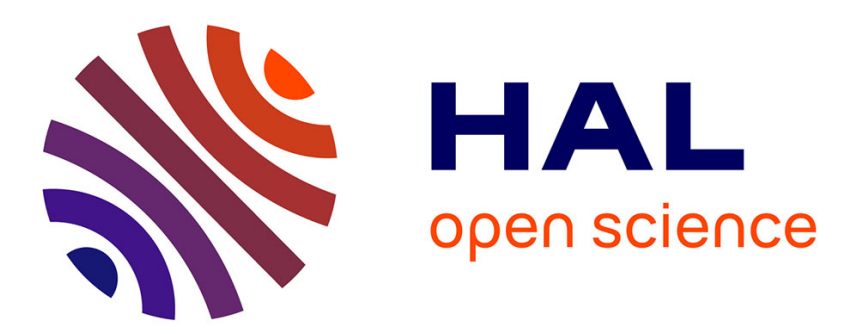

\title{
Experimental analysis of atomic Carbon and Carbon Monoxide production within a high temperature ablative boundary layer
}

Sean Mcguire, Christophe O Laux

\section{- To cite this version:}

Sean Mcguire, Christophe O Laux. Experimental analysis of atomic Carbon and Carbon Monoxide production within a high temperature ablative boundary layer. 55th AIAA Aerospace Sciences Meeting, Jan 2017, Grapevine, France. 10.2514/6.2017-0440 . hal-01866507

\section{HAL Id: hal-01866507 https://hal.science/hal-01866507}

Submitted on 28 Feb 2020

HAL is a multi-disciplinary open access archive for the deposit and dissemination of scientific research documents, whether they are published or not. The documents may come from teaching and research institutions in France or abroad, or from public or private research centers.
L'archive ouverte pluridisciplinaire HAL, est destinée au dépôt et à la diffusion de documents scientifiques de niveau recherche, publiés ou non, émanant des établissements d'enseignement et de recherche français ou étrangers, des laboratoires publics ou privés. 


\title{
Experimental analysis of atomic Carbon and Carbon Monoxide production within a high temperature ablative boundary layer
}

\author{
Sean D. McGuire* and Christophe O. Laux ${ }^{\dagger}$ \\ Laboratoire EM2C, CNRS UPR288, CentraleSupélec, Université Paris Saclay, 92290 Châtenay-Malabry, France
}

\begin{abstract}
Emission measurements in a high temperature boundary layer spanning the ultraviolet to the infrared are presented. The boundary layer is studied after a high enthalpy flow supplied by a plasma torch facility flows around an ablative sample. The experimental configuration is intended to serve as a model of an afterbody flow. The infrared measurements yield estimates of the carbon monoxide ground state concentration present within the boundary layer due to ablation. They also provide a measure of the carbon monoxide rovibrational temperature. These are compared with ultraviolet measurements spanning 190 - $250 \mathrm{~nm}$. Preliminary evidence of $\mathrm{CO}$ 4th positive emission is observed in this spectral region. Atomic carbon emission is observed near the stagnation point, but is weak within the boundary layer due to the relatively low temperature.
\end{abstract}

\section{Introduction}

The design of efficient and reliable heat shields for atmospheric entry continues to be a challenge for space programs looking to pursue sample return, planetary exploration or manned missions within the solar system. Given the severe constraints on mass, the goal is to develop more efficient and lightweight heat shield material. Research is focused on understanding the interaction between a high enthalpy flow and ablative heat shield material in order to identify methods for reducing the heat shield mass and also reduce model uncertainty. The latter point forces engineers to impose large safety margins on the heat shield design which, in turn, leads to a large heat shield mass. Part of the research focus is on the study of forebody conditions, along and around the stagnation streamline. It is also known that afterbody conditions can pose a problem from a shielding standpoint. Johnston and Brandis[1], for example, argue that there may be very significant radiative heat transfer in the afterbody region of a reentering capsule under Stardust conditions. They note, in addition, that ablative species introduced in the forebody have a strong impact on radiative heat transfer within the afterbody, particularly Carbon Monoxide, which was predicted to form in large concentrations within the boundary layer. These conclusions prompted the experimental work done conducted in Ref. [2], which supplied spatially resolved experimental measurements of carbon monoxide concentration in a high temperature ablative boundary layer.

The experimental work done in Ref. [2] used both infrared emission and absorption spectroscopic techniques. One notable conclusion was the absence of measurable quantities of carbon monoxide near the stagnation point. This is to be compared with mole fractions of $\chi_{C O} \approx 0.10$ which were found downstream of the stagnation point. Fig. 1 shows a calculation done with the NASA CEA (chemical-equilibrium with applications, Ref. [3]) code. Given a certain quantity of carbon, the equilibrium calculation shows that atomic carbon and carbon monoxide are the dominant species that form over the wide range of temperatures relevant for reentry flows. This paper describes preliminary measurements of carbon monoxide and atomic carbon, in both the infrared and ultraviolet spectral ranges. Fig. 2 shows the equilibrium densities of some relevant emitting states from ablative species: $\mathrm{CO}(\mathrm{A})$ is responsible for 4 th positive emission while $\mathrm{C}^{1} P^{\circ}$ is responsible for the atomic carbon emission at $248 \mathrm{~nm}$. The goal of this work is to supply experimental measurements which enable a better understanding of the plasma/ablator interaction. For this we use an atmospheric pressure plasma torch facility. A particular advantage of working at atmospheric pressure is that the plasma is in local thermodynamic equilibrium, which enables us to completely characterize the plasma which impinges upon the ablator with a single temperature.[4]

\footnotetext{
* Post-doctoral Researcher, Laboratoire EM2C, CNRS UPR288, CentraleSupélec, Université Paris Saclay, AIAA Member

†Professor, Laboratoire EM2C, CNRS UPR288, CentraleSupélec, Université Paris Saclay, AIAA Associate Fellow
} 


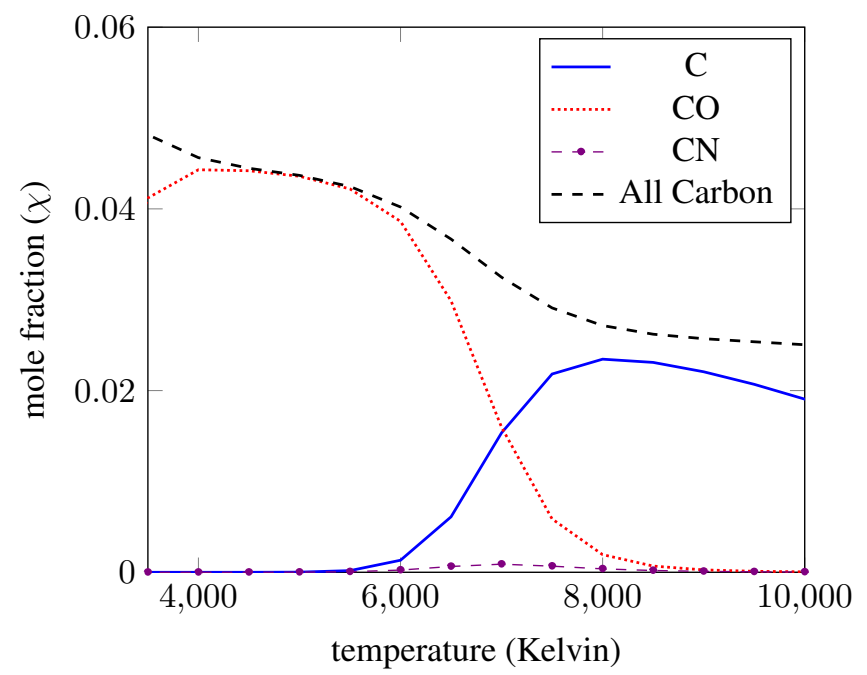

Figure 1. Equilibrium mole fraction of $\mathrm{C}$ and $\mathrm{CO}$ as a function of temperature based upon an input gas mixture of $95 \%$ air and $5 \% \mathrm{C}$ by mole fraction. The curve labeled 'All Carbon' corresponds to the total mole fraction of all carbon-based species taken together $\left(\mathrm{C}, \mathrm{C}_{2}, \mathrm{CN}, \mathrm{CO}\right.$ and their respective ions). $\mathrm{C}$ and $\mathrm{CO}$ constitute the primary ablative species over the temperature range plotted here. Ionic carbon $(\mathrm{C}+)$ becomes important above $7500 \mathrm{~K}$ and accounts for the residual at high temperatures.

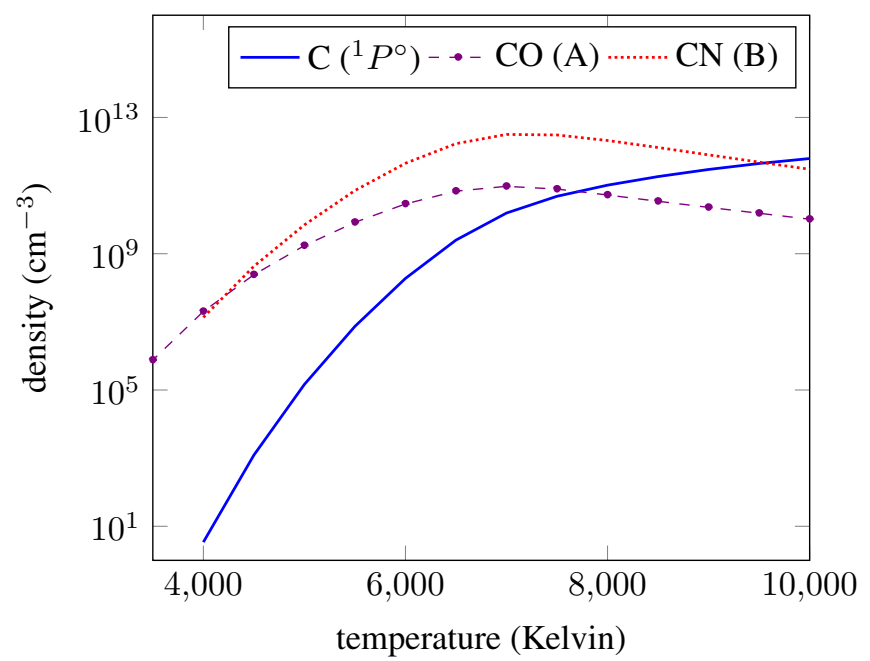

Figure 2. Absolute densities of some relevant emitting states from ablative species assuming thermochemical equilibrium.

\section{Experimental Setup}

The plasma torch facility used is a TAFA Model 66 inductively coupled plasma (ICP) torch powered by a 120 kVA radio frequency LEPEL Model T-50-3 power supply. The power supply operates at $4 \mathrm{MHz}$ and can supply a maximum of $12 \mathrm{kV}$ DC and $7.5 \mathrm{~A}$ to the oscillator plates. Details of the plasma torch facility are provided in previous publications: for example, see MacDonald et al.[5, 6, 7] For the experiments presented here, a 2-cm diameter exit nozzle was used. The characteristics of the plasma produced by the torch facility with this $2 \mathrm{~cm}$ exit nozzle have been previously studied and documented in Ref. [5]. In particular, the centerline temperature and velocity are approximately $6500 \mathrm{~K}$ and $140 \mathrm{~m} / \mathrm{s}$, respectively. Figure 3 shows a temperature profile of the plasma, measured without a sample placed in the plasma and taken $1.5 \mathrm{~cm}$ downstream of the nozzle exit. The temperature was measured from the absolute emission of the atomic oxygen line at $777 \mathrm{~nm}$ and the procedure is documented in Ref. [4, 8]. For the tests in this paper, a cylindrical sample of carbon preform (Mersen, CBCF 18-2000) was placed in the plasma flow. The sample has a diameter of approximately $1.5 \mathrm{~cm} .{ }^{\text {a }}$ The sample position relative to the plasma flow is shown in Fig. 4 .

Fig. 5 shows the optical layout used for the experiments. Quantitative emission spectroscopy was used for the

${ }^{\mathrm{a}}$ This is to be compared with a diameter of $2 \mathrm{~cm}$ in Ref. [2]. 


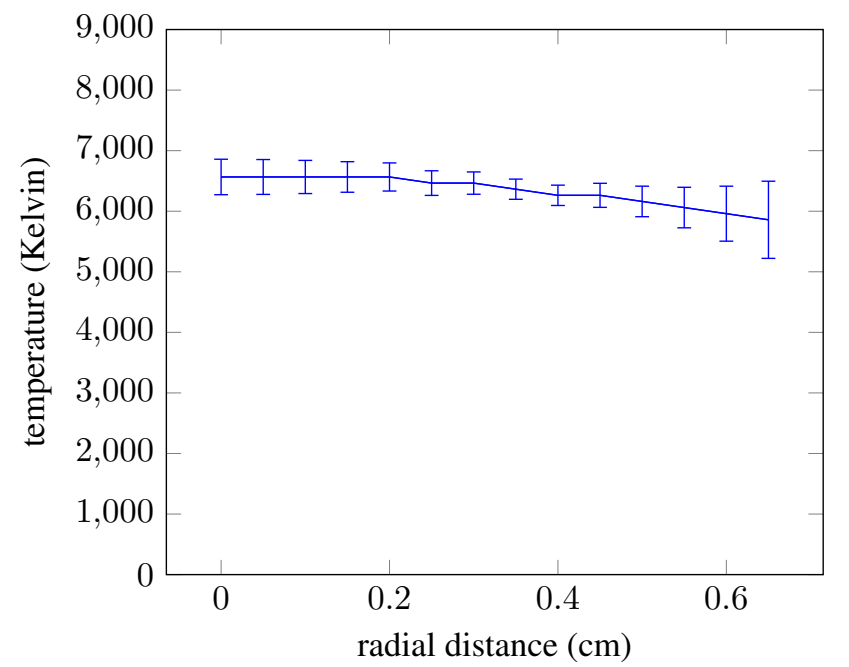

Figure 3. Plasma temperature profile measured $1.5 \mathrm{~cm}$ above nozzle exit from the absolute intensity of the atomic oxygen triplet at $777 \mathrm{~nm}$. The measurement was done with no sample inserted into the flow and $r=0$ corresponds to the flow centerline.

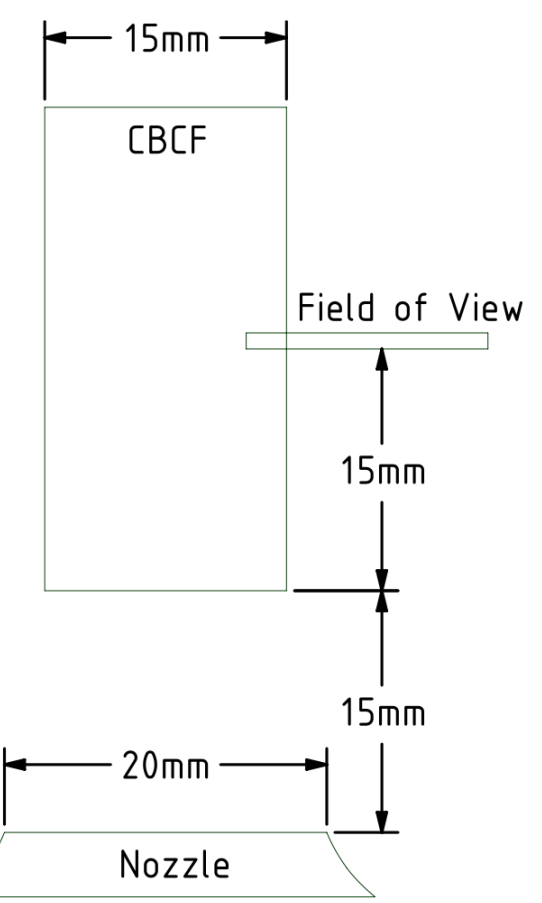

Figure 4. Relative positions of CBCF sample, torch nozzle and spectrometer field of view. The spectrometer field of view is approximately $1 \mathrm{~cm}$ long. In this diagram, the plasma exits the nozzle vertically and impinges upon the CBCF sample.

measurements. Two spectrometers were used, one in the ultraviolet for tracking the emission of $\mathrm{C}$ and $\mathrm{CO}$ 4th positive. The second spectrometer was an infrared system used for tracking infrared Carbon Monoxide emission within the electronic ground state and centered around $4.6 \mu \mathrm{m}$. The infrared emission setup for tracking carbon monoxide is essentially that detailed in Ref. [2]. There, both emission and absorption measurements were taken simultaneously and utilized the $\Delta \nu=1$ vibrational transitions within the ground $\mathrm{X}^{1} \Sigma^{+}$electronic state. Three spectral features located in the vicinity of $4.6 \mu \mathrm{m}$ were used for quantitative measurements: peak ratios were used as a measure of temperature while absolute magnitudes were used for concentration measurements. These features are sensitive to temperature over the range of interest $(3000-7000 \mathrm{~K})$ as they incorporate emission from rotational levels $J=7-46$ and vibrational levels $\nu=1-5$. For the experiments in this paper, only emission measurements are used because these were found to have better signal-to-noise characteristics than absorption measurements under our experimental 


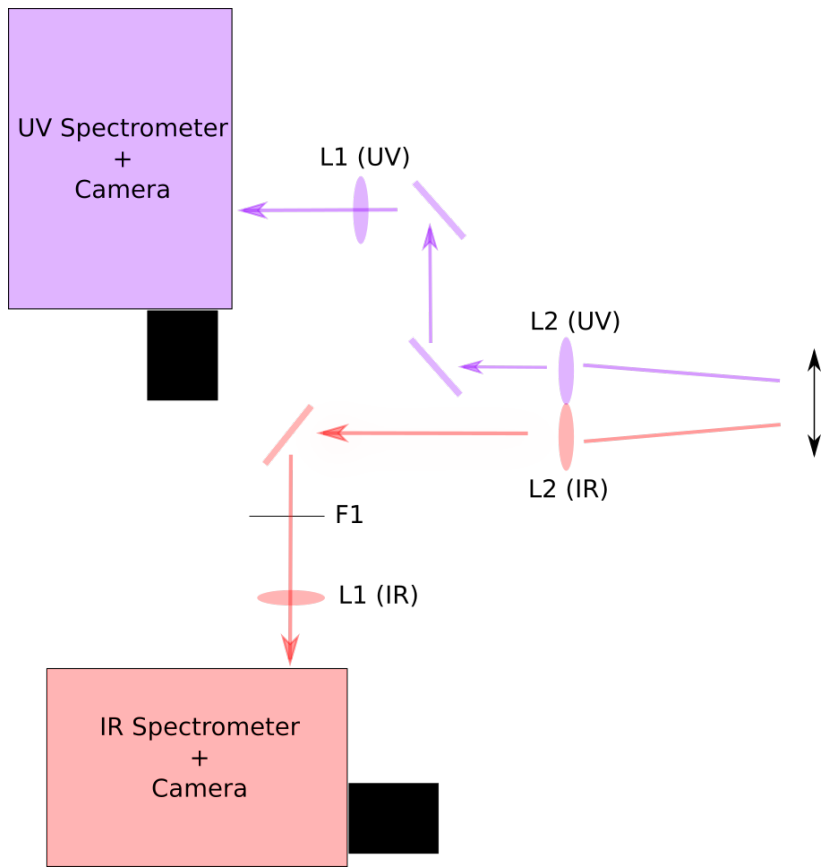

Figure 5. Optical layout used for emission measurements: L2 (UV) $=$ L2 (IR), L1 (UV) $=$ L1 (IR). F1 is a high pass filter to suppress interference from other diffraction orders within the infrared spectrometer. Both spectrometer slits are imaged onto the same spot, denoted by the arrow. They are imaged such that the slit image is horizontal and occupies the field of view defined in Fig. 4. To ensure that both systems were imaged onto the same point, both ultraviolet and infrared images of a globar material were taken and the system aligned based upon these images.

conditions. For the emission measurements, an infrared camera is mounted at the exit of the infrared spectrometer for imaging. The spectrometer is a SpectraPro 2750 from Princeton Instruments. The grating is blazed at $4.0 \mu \mathrm{m}$ and has 300 grooves $/ \mathrm{mm}$. At the output of the spectrometer, an IRC 800 infrared camera from Infrared Cameras was mounted and used with an integration time of $450 \mu s$. 300 shots were averaged over a 1.5 second interval to form a single image used for spectral analysis. Note, also, that a high pass filter with a cut-off wavelength of $3.6 \mu \mathrm{m}$ was placed in the optical path to prevent higher diffraction orders within the spectrometer from interfering with the measurements of $\mathrm{CO}$ emission at $4.6 \mu \mathrm{m}$ : particularly, in order to suppress $\mathrm{CO}$ emission at $2.3 \mu \mathrm{m}$. For ultraviolet measurements, an ultraviolet spectrometer and camera were used. The spectrometer was a SpectraPro 2500i from Princeton Instruments with a grating blased at $300 \mathrm{~nm}$ with 600 grooves $/ \mathrm{mm}$. The camera was a UV/VUV enhanced Pi-Max camera from Princeton Instruments. Integration times between 1 and $50 \mathrm{~ms}$ were used depending on the measurement. 5 shots were averaged over a 1.5 second interval to form a single image for spectral analysis. For absolute intensity calibration in the infrared, a calibrated tungsten lamp with Sapphire output window was used. For the ultraviolet region, a calibrated Argon discharge was used. Both are traceable to NIST standards. Finally, to ensure that measurements were taken at the same location relative to the sample, two images were taken of a globar material, one with each system. In both cases, the spectrometer slits were completely opened and the grating tuned to $\lambda=0$ to act as a mirror. The globar material is a strong infrared emitter but also emits at ultraviolet wavelengths. The optics were adjusted to ensure that both systems imaged the same region of space. During tests, each spectrometer was tuned to its respective wavelength and the image captured via each camera was used to both spatially and spectrally resolve the emission. The infrared system was used to track the location of the ablative sample surface as the continuum emission was easily visible in the infrared but not in the ultraviolet. Once the sample surface position was identified with the infrared measurements, it could then be marked within the ultraviolet measurements.

The slit function for the infrared measurements was measured to be trapezoidal with an equivalent FWHM of 1.6 $\mathrm{nm}$. The slit function for the ultraviolet measurements was measured to be trapezoidal with an equivalent FWHM of $0.3 \mathrm{~nm}$. 


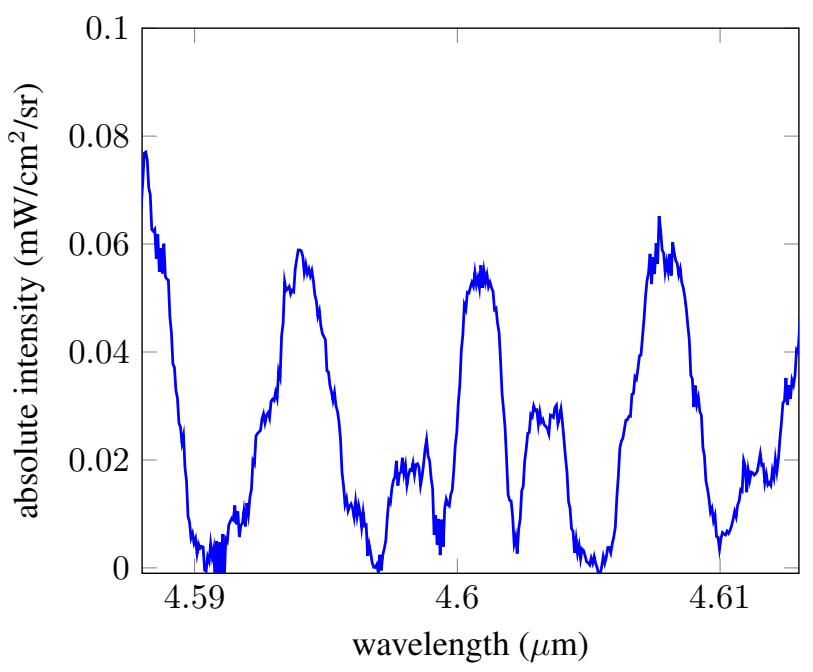

Figure 6. Carbon Monoxide spectrum generated by binning across the boundary layer.

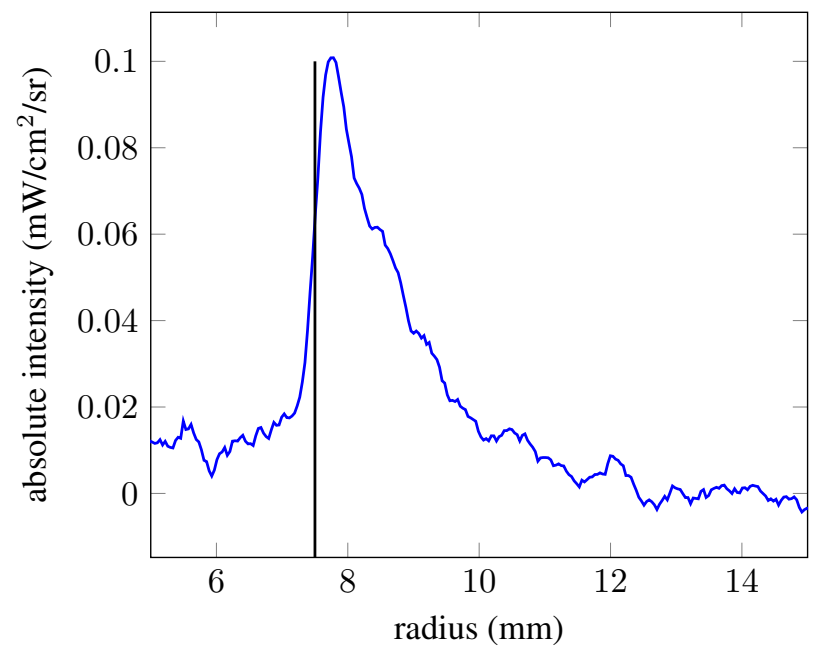

Figure 7. Measured spatial dependence of the carbon monoxide. The emission from the central peak at $4.6 \mu$ is plotted as a function of radius. The sample surface is located at $7.5 \mathrm{~mm}$.

\section{Results \& Discussion}

\section{III.A. infrared measurements}

Figure 6 shows a sample infrared spectrum taken approximately 3 seconds after sample insertion. The spectrum was obtained by binning across the boundary layer. In Ref. [2] the signal-to-noise ratio of the carbon monoxide measurements was larger due to the bigger sample diameter used for experiments. This permitted a full spatial analysis of the carbon monoxide emission signal. For these experiments, a smaller sample diameter was chosen to be less than the nozzle diameter in order to ensure as much as possible that the sample was exposed to a uniform flow. However, this caused a drop in the signal-to-noise ratio of the emission measurements. Due to the smaller sample diameter, less material is in contact with the flow and less $\mathrm{CO}$ is produced. The optical path length associated with the emission measurements is also smaller (see Fig. 9). The combined effect is to reduce the signal-to-noise ratio of the measurement and this drop was sufficient that a fully spatially resolved analysis was not possible with the sample diameter chosen for these experiments. To obtain quantitative estimates, some assumptions were made. The measured spatial distribution in Fig. III.A was replaced by a uniform distribution. For this, the average distance away from the sample surface was first calculated: 


$$
l=\frac{\int \Delta r \chi(r) d r}{\int \chi(r) d r}
$$

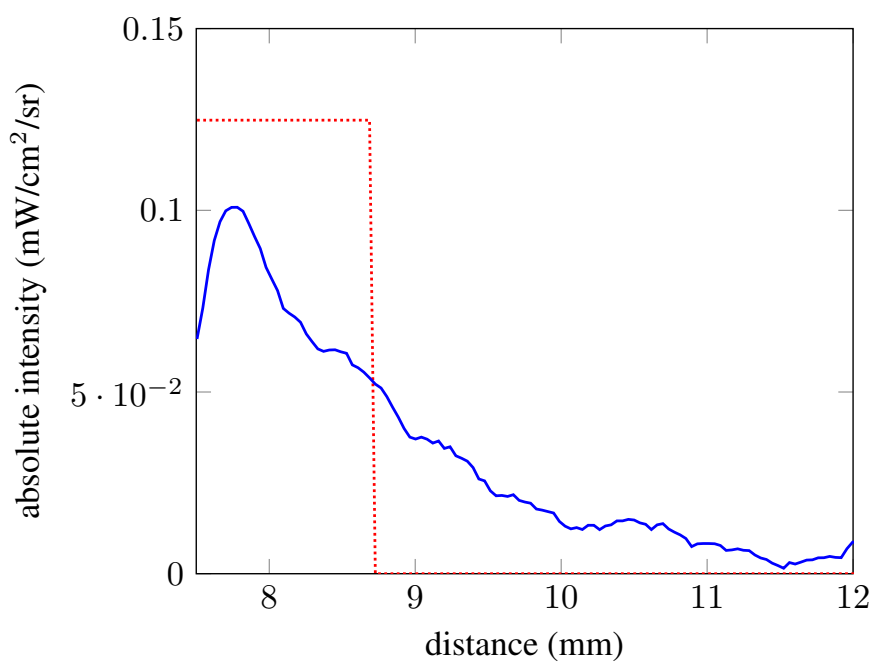

Figure 8. The measured distribution of carbon monoxide (blue/solid) is replaced by a uniform distribution (red/dashed). The uniform distribution was constructed by calculating the average distance away from the sample surface: the distribution was assumed constant across this distance and the magnitude was calculated such that the area under the two curves is the same.

where $\Delta r$ is the distance from the sample surface. This yielded an average value of $l \approx 1.2 \mathrm{~mm}$. The magnitude of the uniform distribution was then chosen to conserve the total quantity of carbon monoxide: i.e. the magnitude was chosen such that the area under the real distribution was equal to the area under the uniform distribution. Figure 8 illustrates.

Figure 9 shows the orientation of the optical path with respect to the sample surface. Note that $l$, as calculated in Eqn. 1 represents a measure of the distribution of carbon monoxide away from the sample surface, but does not represent the relevant optical path length. To estimate the optical path length, the geometrical calculation shown in Fig. 9 is used and yields a value of approximately $9 \mathrm{~mm}$. With this path length estimate, the theoretical emission spectra shown in Fig. 10 could be calculated by varying the mole fraction of carbon monoxide: the best fit resulted is a mole fraction of $\chi_{C O}=0.06$.

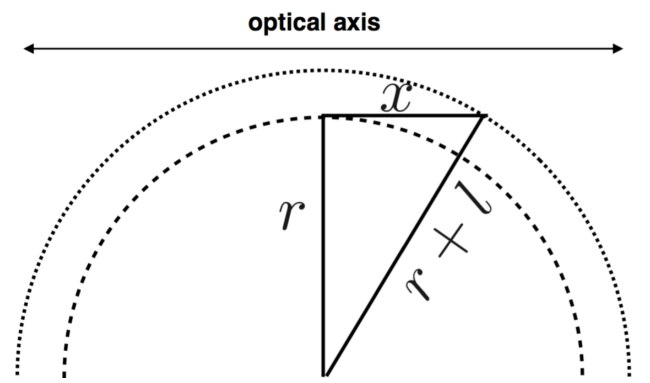

Figure 9. Diagrams illustrating the estimate of the optical path length. Given a sample radius of $r=7.5$ mm and a carbon monoxide distribution of $l=1.2$ $\mathbf{m m}$, the optical path length is approximately $2 x=2 \sqrt{(r+l)^{2}-r^{2}} \approx 9 \mathbf{m m}$.

The overall picture given by the IR measurements of the boundary layer indicates that we have an average CO mole fraction of $\chi_{C O} \approx 0.06$ at an average temperature of about $5000 \mathrm{~K}$. Furthermore, given the spatial distribution of the signal, we estimate that this CO persists approximately $2-3 \mathrm{~mm}$ away from the sample surface (see Fig. 8). 


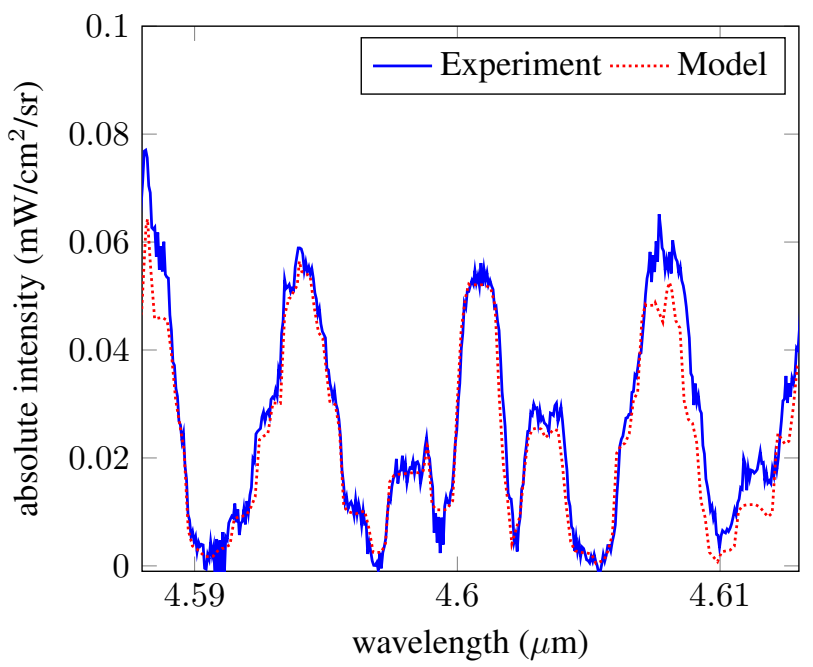

Figure 10. Experimental spectra as compared to a model spectrum generated with SPECAIR. The model spectrum was generated by assuming an optical path length of $l=0.9 \mathrm{~cm}, T=5000 \mathrm{~K}( \pm 500 \mathrm{~K})$ and $\chi_{C O}=0.06( \pm 0.05)$. The small discrepancies in the spectra are because the experimental spectrum was averaged across a relatively wide range of temperatures from the sample surface to the edge of the boundary layer. The artificial drop-off in the experimental spectrum at the edges is where the spectral collection window stops.

\section{III.B. ultraviolet measurements}

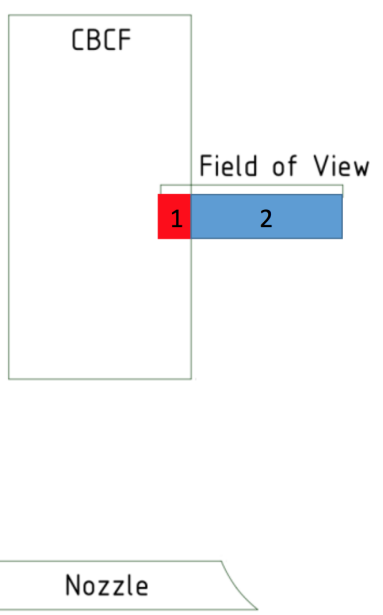

Figure 11. For analysis, spectra were binned over the spatial dimensions indicated in this figure. 1 represents the portion of the spectra observed on sample. 2 represents the boundary layer. For spectra taken before sample insertion, the spectra were binned over the entire spatial domain.

To explain the general trends in the ultraviolet data, spectra are binned over the spatial domains indicated in Fig. 11. Note that, even prior to binning, the spectra observed at a particular point is a line-of-sight integrated value. Thus these binned measurements represent average spectra. Beginning with the boundary layer (region 2 in Fig. 11), Fig. 12 shows a binned spectrum taken just after sample insertion. Note the presence of strong atomic lines identified in the figure. We attribute these to ionic sodium emission which has been noted in other ablation experiments before: see, for example, MacDonald et al.[7]. Figure 13 compares spectra produced from regions 1 and 2 identified in Fig. 11: it is quite apparent that when looking on sample, the spectra are much more intense. This was a general trend observed in the experiments and suggests that there is a thin surface layer which contributes very intense emission to the signal. These initial spectra presented in Figs. 12 and 13 were produced approximately 3 - 5 seconds after sample insertion. This point in time was chosen so as to minimize the change in sample geometry due to ablation while also providing sufficient time for the emission to stabilize after sample insertion. Beyond this time, the sample geometry begins to morph due to ablation, particularly beyond approximately 10 seconds. Nonetheless, qualitative observations may still be presented. Beginning with the boundary layer emission (i.e. region 2 in Fig. 11); as the stagnation point recedes towards the field of view, the sodium emission is observed to decrease, leaving just high temperature air emission. The 


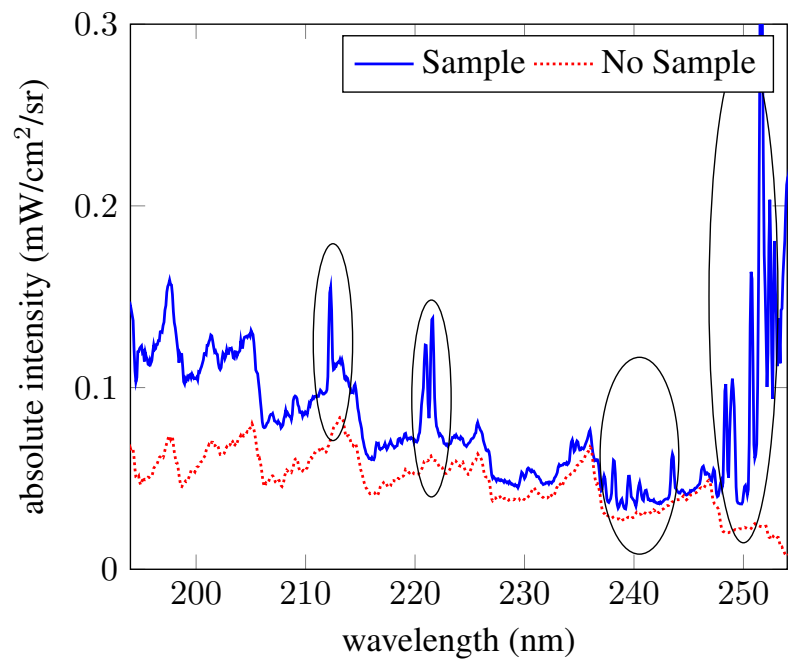

Figure 12. Ultraviolet spectra, before (No Sample) and after (Sample) sample insertion. Note that the 'No Sample' spectrum does not correspond to the centerline spectrum of the plasma torch as the field of view is placed off center. The boundary layer spectrum was taken about 3 seconds after sample insertion: early enough that the sample geometry had not changed substantially but late enough that initial transients have died off. The circled features are believed to come from sodium emission.

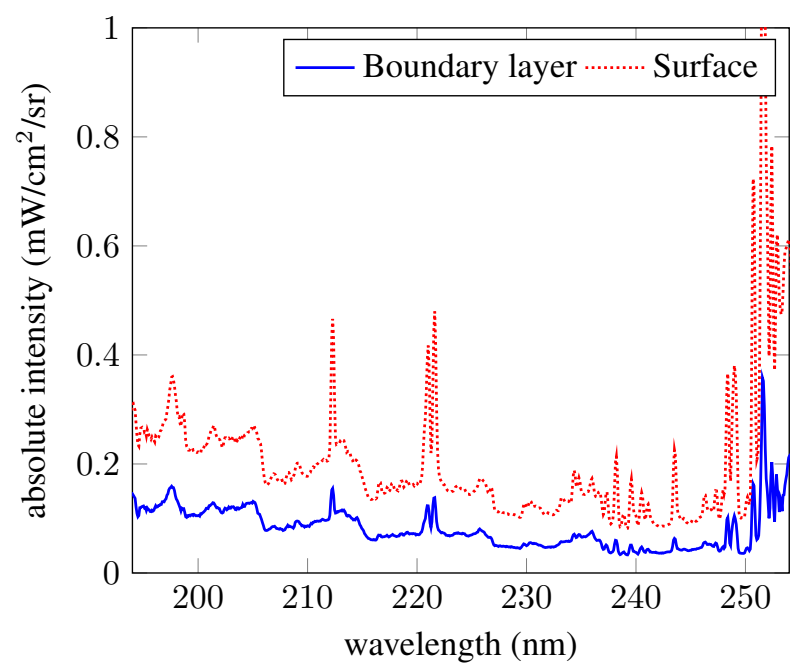

Figure 13. Comparison of spectra obtained while looking in the boundary layer (region 2 in Fig. 11) and on the sample surface (region 1 in Fig. 11).

trends are generally the same when binning over the on sample emission (i.e. region 1 in Fig. 11) with one exception. As the stagnation point recedes through the field of view, we observe atomic carbon emission. Figure 14 illustrates one such spectrum taken near the stagnation point. This observation of atomic carbon emission is consistent with other stagnation point measurements: see, for example, Ref. [9].

Returning to the boundary layer emission presented in Fig. 12, we expect to see UV/VUV 4th positive emission due to the presence of Carbon Monoxide, identified by the infrared measurements. A few factors complicate definitive identification of this emission. First, an independent, spatially resolved measure of the gas temperature is not available within the boundary layer. Only the estimate given by the $\mathrm{CO}$ measurements and the initial free stream measure of 6500 Kelvin are available. Second, aside from the ionic sodium emission, the spectral emission is a complex mixture of molecular bands. The high temperature air emission over this wavelength region is a mixture of NO bands $(\beta, \Delta$, $\epsilon$ and $\gamma$ ). Figure 15 shows a theoretical air emission spectrum generated with the software package SPECAIR[8, 10] for an air temperature of $5500 \mathrm{~K}$ and Carbon Monoxide 4th positive emission for three different temperatures for comparison. If, for example, we assume a Carbon Monoxide temperature of $5000 \mathrm{~K}$ and look at the combined Carbon Monoxide and air emission shown in Fig. 16, there is a limited set of features that distinguish this emission from air emission. A close look at Fig. 12 indicates that, indeed, these features may be present in the experimental spectra. 


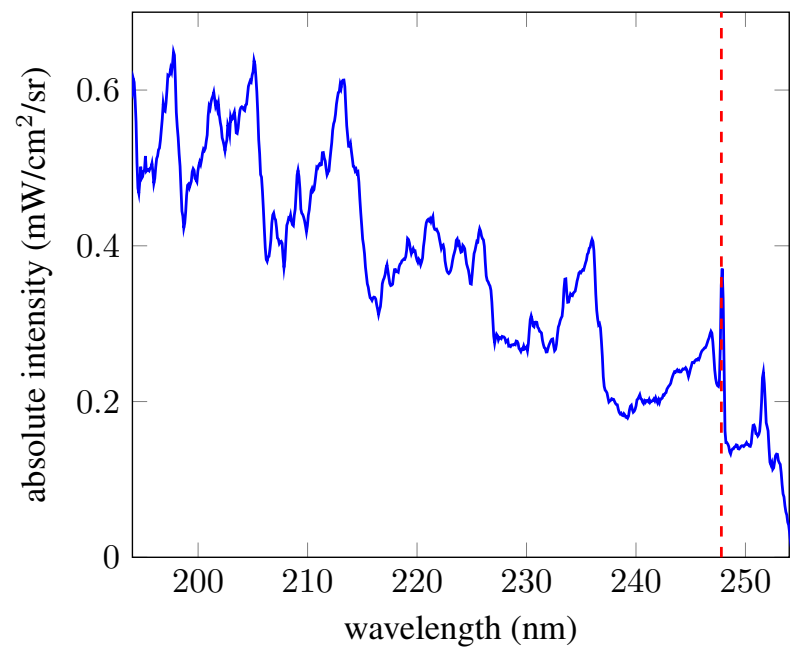

Figure 14. Ultraviolet spectra taken near the stagnation point and showing the atomic Carbon emission observed at $248 \mathrm{~nm}$.

This cannot be said definitively, however. In an attempt to improve the identification of these features, the boundary layer spectra were Abel inverted. Figure 17 shows the Abel inverted spectra located just beyond the sample surface. Again, as before, the 4th positive features appear to be present but are not strong enough to be definitively identified as such.

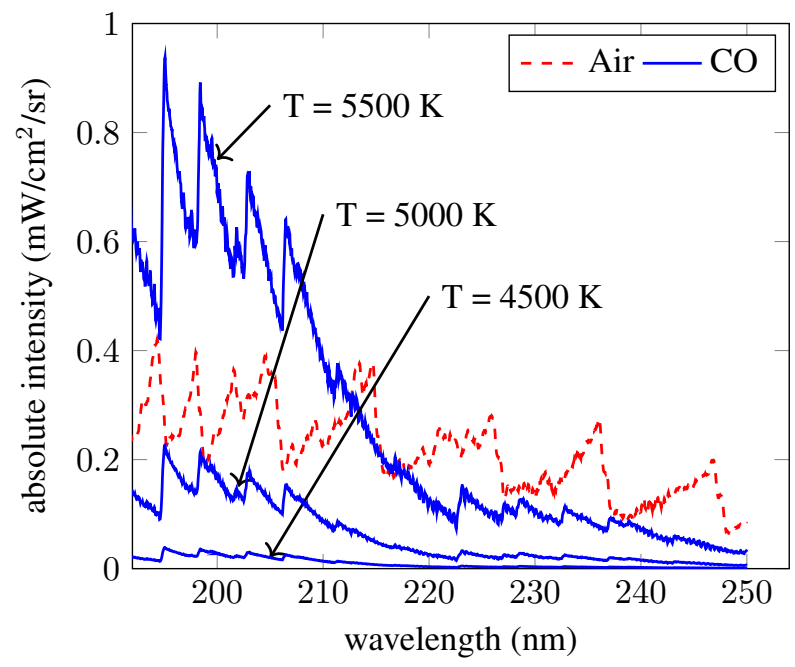

Figure 15. Theoretical equilibrium air emission from a $1 \mathrm{~cm}$ plasma slab at a temperature of 5500K (red,dashed). Carbon Monoxide 4th positive emission (solid/bue) for a mole fraction of $5 \% \mathrm{CO}$ at three separate temperatures: $4500 \mathrm{~K}, 5000 \mathrm{~K}$ and $5500 \mathrm{~K}$. These plots are generated using the SPECAIR software.

\section{Conclusion}

We report infrared $(4.6 \mu \mathrm{m})$ and ultraviolet $(190-250 \mathrm{~nm})$ emission measurements in the high temperature ablative boundary layer around a carbon preform ablative sample. The infrared measurements targeted the electronic ground state CO density. At the side of the ablative sample, the mole fraction of $\mathrm{CO}$ is about $5 \%$ and its temperature is approximately $5000 \mathrm{~K}$. This is consistent with the spatially resolved measurements reported in Ref. [2]. In contrast, no $\mathrm{CO}$ was detected at the stagnation point. The ultraviolet measurements were conducted to detect $\mathrm{CO} 4$ th positive and atomic carbon emission. Carbon is clearly observed near the stagnation point but was found to be much weaker in the boundary layer at the side of the sample. No CO 4th positive emission is observed at the stagnation point, but may be present on the sample side. Further measurements are required underway to confirm the presence of $\mathrm{CO} 4$ th positive emission. These results suggest that the carbon produced at the stagnation point recombines to produce $\mathrm{CO}$ 


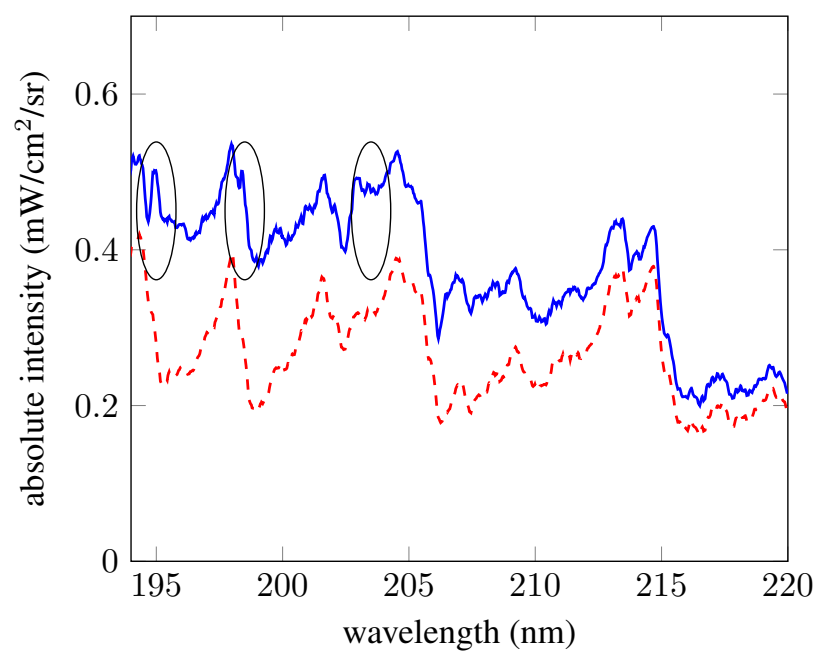

Figure 16. Combined emission from Carbon Monoxide 4th positive emission at $5000 \mathrm{~K}$ and equilibrium air emission at $5500 \mathrm{~K}$ (blue). Equilibrium air emission at $5500 \mathrm{~K}$ (red/dashed) is shown for comparison. Some features which distinguish the CO/air mixture emission from pure air emission are circled.

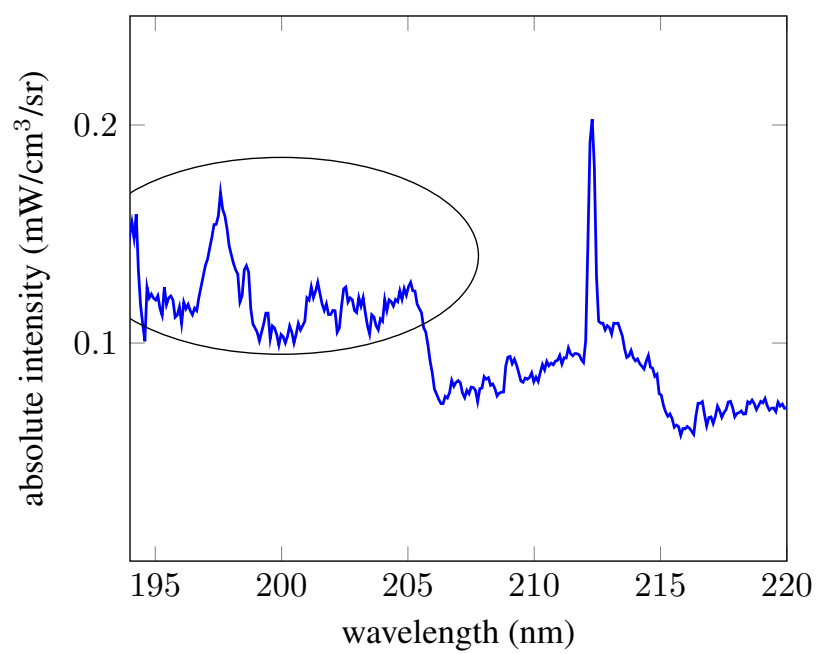

Figure 17. Experimental spectra obtained after Abel inverting the experimental spectra in the boundary layer. The circled regions are supposed to contain 4th positive emission, though they are not distinct enough to definitively identify as CO 4th positive emisison.

as it flows around the ablative sample.

\section{References}

1 Christopher O. Johnston and Aaron M. Brandis. Features of afterbody radiative heating for earth entry. Journal of Spacecraft and Rockets, 52(1):105-119, 2016/07/26 2014.

2 S D McGuire, A C Tibère-Inglesse, and C O Laux. Infrared spectroscopic measurements of carbon monoxide within a high temperature ablative boundary layer. Journal of Physics D: Applied Physics, 49(48):485502, 2016.

3 B. J. McBride and S. Gordon. Computer program for calculating and fitting thermodynamic functions. Technical report, NASA RP-1271, 1992.

${ }^{4}$ Christophe O. Laux. Optical Diagnostics and Radiative Emission of Air Plasmas. PhD thesis, Stanford University, Dept. of Mechanical Engineering, 1993.

5 Megan MacDonald and Christophe O. Laux. Experimental characterization of ablation species in an air plasma ablating boundary layer. In 11th AIAA/ASME Joint Thermophysics and Heat Transfer Conference, number AIAA2014-2251. American Institute of Aeronautics and Astronautics, June 2014. 
${ }^{6}$ M. E. MacDonald, C. M. Jacobs, and C. O. Laux. Interaction of air plasma with ablating heat shield material. IEEE Transactions on Plasma Science, 42(10):2658-2659, Oct 2014.

7 Megan E. MacDonald, Carolyn M. Jacobs, Christophe O. Laux, Fabian Zander, and Richard G. Morgan. Measurements of air plasma/ablator interactions in an inductively coupled plasma torch. Journal of Thermophysics and Heat Transfer, 29(1):12-23, 2014.

8 C O Laux, T G Spence, C H Kruger, and R N Zare. Optical diagnostics of atmospheric pressure air plasmas. Plasma Sources Science and Technology, 12(2):125, 2003.

9 Tobias Hermann, Stefan Loehle, Pénélope Leyland, Lionel Marraffa, Jean-Marc Bouilly, and Stefanos Fasoulas. First results on ablation radiation coupling through optical emission spectroscopy from the vacuum ultraviolet to the visible. In European Symposium on Aerothermodynamics for Space Vehicles, 2015.

10 SPECAIR, Software Package, Ver. 3.0, SpectralFit S.A.S., http://www.spectralfit.com. 